Hence the $C^{*}$-algebra $\pi_{\tilde{\chi}}(A)$ and so $A$ have a type III-factor *-representation.

This completes the proof.

\title{
REFERENCES
}

1. J. W. Calkin, Two sided ideals and congruences in the ring of bounded operators in Hilbert space, Ann. of Math. 42 (1941), 839-873.

2. J. Dixmier, Les algebres d'operateurs dans l'espace hilbertien, Gauthier-Villars, Paris, 1957.

3. J. Glimm, Type I $C^{*}$-algebras, Ann. of Math. 73 (1961), 572-612.

4. I. Kaplansky, The structure of certain operator algebras, Trans. Amer. Math. Soc. 70 (1951), 219-255.

5. S. Sakai, On topological properties of $\mathrm{W}^{*}$-algebras, Proc. Japan Acad. 33 (1957), 439-444.

6. ——, The theory of $W^{*}$-algebras, Lecture notes, Yale University, 1962.

7. _- - On a problem of Calkin, Amer. J. Math. (to appear).

8. J. Schwartz, Two finite, non-hyperfinite, nonisomorphic factors, Comm. Pure Appl. Math. 16 (1963), 19-26.

9. - Non-isomorphism of a pair of factors of type III, Comm. Pure Appl. Math. 16 (1963), 111-120.

University of Pennsylvania

\section{SOME UNSYMMETRIC COMBINATORIAL NUMBERS}

\section{BY ANDREW SOBCZYK}

Communicated by V. Klee, January 26, 1966

By an $n$-configuration we shall mean an abstract set of $n$ elements, together with the set of all unordered pairs of distinct elements from the set. It is convenient also to use quasi-geometrical terminology such as vertex for element, edge or side for a pair (2-tuple), triangle as well as triple (3-tuple) for a 3-subconfiguration, and so on.

The Ramsey number $N(p, q, 2)$ (see [3, pp. 38-43], or [2, pp. 61$65]$ ), for two kinds $h, v$ of pairs (or two "colors of edges"), is the smallest integer such that if $n \geqq N(p, q, 2)$, then any $n$-configuration is sure to contain either an $h p$-tuple (a $p$-tuple all of whose edges are $h)$ or a $v q$-tuple. Call a $p$-tuple all of whose edges are alike $(h$ or $v)$ a like $p$-tuple. We introduce, and partially determine the values of, new analogous combinatorial numbers $K(p, q, 2), M(p, q, 2)$, and $V(p, q, 2)$.

Definitions. The number $K(p, q, 2)$ is the smallest integer such that if $n \geqq K(p, q, 2)$, then for each vertex, the configuration is sure to contain either a like $p$-tuple containing the vertex, or a like $q$-tuple not containing the vertex. For three kinds $r, g, v$ of edges, $M(p, q, 2)$ 
is the smallest integer such that if $n \geqq M(p, q, 2)$, the configuration is sure to contain either a like $p$-tuple, or a $j, k q$-tuple (a $q$-tuple having at most two kinds $j, k$ of edges, where $j, k=r, g$, or $v)$. The number $V(p, q, 2)$ is the smallest integer such that if $n \geqq V(p, q, 2)$, then for each vertex of the configuration, the configuration contains either a like $p$-tuple containing the vertex, or a $j, k q$-tuple not containing the vertex.

Consider for a moment "verticial" numbers, which otherwise are like the Ramsey numbers: $S(p, q, 2)$, for example, is the smallest integer such that a configuration with $n \geqq S(p, q, 2)$ is sure to contain, for each vertex, either an $h p$-tuple containing the vertex, or a $v$ $q$-tuple not containing the vertex. Evidently $N(p, q, 2) \leqq S(p, q, 2)$. But for all $p \geqq 3, q \geqq 3, S(p, q, 2)=\infty$ : for arbitrarily large $n$, at one vertex, assign $(p-1)$ edges from the vertex to be $h$, the remainder $v$. Let one edge joining a pair of other ends of the $(p-1)$ edges be $v$, and let all other edges of the $n$-configuration be $h$. Then for the vertex, the $n$-configuration contains neither an $h p$-tuple containing the vertex, nor a $v q$-tuple not containing the vertex. Moreover $S(p, q, 2)=\infty$ for all $p \geqq 2, q \geqq 2$.

Denote by $W(q, p, 2)$ the smallest in teger such that if $n \geqq W(q, p, 2)$, then for each vertex the configuration is sure to contain either a $j, k q$-tuple containing the vertex, or a like $p$-tuple not containing the vertex. We notice that $W(q, p, 2)=V(p, q, 2)$.

Our results so far concerning the numbers $K, M, V$ are indicated in the following Theorem 1 (including the table) and Theorem 2. For purposes of comparison, the known values of the Ramsey numbers $N$ also are included: the entries in the table are the values of $N, K, M, V$ in that order, for each $p, q$.

Theorem 1. For $p, q$ from 3 to 5 inclusive, the numbers have values as given in the following table (cf. the table in [3, p. 42]).

\begin{tabular}{|c|c|c|c|}
\hline$P$ & 3 & 4 & 5 \\
\hline 3 & $6,6,5,6$ & $9,8,8,10$ or 11 & $14,10,14,14$ \\
\hline 4 & $9,7,5,6$ & $18,18,10$ to 17,11 to 18 & \\
\hline & $14,7,5,6$ & & \\
\hline
\end{tabular}

For all $p>3$, we have that $K(p, 3,2)=7$; for all $q \geqq 3$, that $K(q, q, 2)$ $=N(q, q, 2)$ and $K(3, q, 2)=2 q ;$ for $p>q$, that $K(p, q, 2) \leqq N(q, q, 2)+1$; and for $q>p>3$, that $K(p, q, 2) \geqq \max (K(p-1, q, 2)+1,2 q+p-3)$. Further, $N(3,6,2)$ is 17 or 18 (cf. [1]), and $17=M(3,6,2) \leqq N(3,6,2)$. 
A configuration is called degenerate, with respect to any of the combinatorial numbers, in case it does contain (for each vertex in case of $V)$ either a $p$-tuple or a $q$-tuple as described in the corresponding definition. As an example, it is quite easy to find an 8-configuration which is nondegenerate with respect to $N(3,4,2)$-an octagon with an 8-cycle plus a 4-cross (see below) of blue edges, and an 8-cycle plus two 4-cycles of red edges, has neither a blue triangle nor a red quadruple (cf. the existence proof for the 8-configuration in [1]). The method of establishing the above lower bounds $L$ is to exhibit in each case a nondegenerate configuration with $n=L-1$. To establish an upper bound $U$, it is sufficient to show that any configuration with $n=U$ must be degenerate. The value of a combinatorial number of course is determined in case $L=U$. Details will be included in a paper which will be offered for publication elsewhere.

A subsidiary result, analogous to Steiner triple systems ([2] or [3]), is the following. A $k$-cycle is a closed string of $k$ successively adjacent edges, such as $12 ; 23 ; \cdots ; k-1, k ; k, 1$; where $\{1, \cdots, k\}$ is a subset of $k$ of the vertices of the configuration. In any $(2 n+1)$ configuration, the edges can be covered (each exactly once) by $n k$-cycles with $k=(2 n+1)$. A $k$-cross is a set of $k$ edges, no two of which are adjacent. The edges of any $(2 n+2)$-configuration can be covered by $n(2 n+2)$-cycles and an $(n+1)$-cross.

Theorem 2. We have $K(p, q, 2) \leqq N(p, q, 2), M(p, q, 2) \leqq N(p, q, 2)$, $M(p, q, 2) \leqq V(p, q, 2)$. For each $q, V(q, q, 2)$ is either $M(q, q, 2)$ or $M(q, q, 2)+1 ;$ for any $p, V(p, q, 2) \leqq M(q, q, 2)+1$. For $p>q$, $M(q, q, 2) \leqq \min (M(p, q, 2), V(p, q, 2))$, and $V(q, q, 2) \leqq V(p, q, 2)$. For $p \geqq 3, M(p, 3,2)=5$, and $V(p, 3,2)=6$. For $q>3, V(3, q, 2)$ $\leqq(3 q-1)$.

Reference [4], for example, indicates the wealth of possible applications for combinatorial results.

\section{REFERENCES}

1. A. M. Gleason and R. E. Greenwood, Combinatorial relations and chromatic graphs, Canad. J. Math. 7 (1955), 1-7.

2. Marshall Hall, Jr., A survey of combinatorial analysis, Some aspects of analysis and probability, Wiley, New York, 1958, pp. 35-104.

3. H. J. Ryser, Combinatorial mathematics, Carus Monograph, No. 14, Math. Assoc. of America, Wiley, New York, 1963.

4. R. G. Busacker and T. L. Saaty, Finite graphs and networks, McGraw-Hill, New York, 1965.

Clemson University 